\title{
List of reviewers
}

JOURNAL OF ADVANCES IN MATHEMATICS (2015).

The editorial team of the journal would like to thank the following reviewers for their work in referring manuscripts during 2015.

Sana Abughurra

Mohammad Javad Ebadi

Ankur Singh Bist

W. S. Amer

Ayyappan Govindasamy

Emilio Ram

Juid

Behnamf Razzaghf

Faizan Ahmed

Najah Ali Al-Ziadi

Ajaz Ahmad Dar

Muaadh Almahalebi

Rauhi I. Elkhatib

Kimuya M Alex

Fethi Bin Muhammad

Belgacem

Dr. Ramesh Prasad Aharwal

Shimaa A. Dessoky

Selami Ercan
Professor Zoran D Mitrovia

Mustafa Ozkan

Om Fatima Doaebil

Mustafa Turkyilmazoglu

Cesar Arturo Garza

Jeffrey Boyd

Murad Abuamr

Saratha Sathasivam

Shukur Neamah Al-Aeashi

Meltem Erden Ege

Maria Alessandra Ragusa

Vinodh Kumar Kumar

Chellamuthu

Fernane Khaireddine

Youssef El Foutayeni

Elena Corina Cipu

Shanmuga Sundaram O.V

Antonio Nicola Di Teodoro

Shalini Shekhawat
Mustafa Bilici

Quinn Jackson

Hasan

Inci Albayrak

Zhang Xi Wen

$\mathrm{K}$ Thirusangu

Erjola Cenaj

Muhammad Kamil Abidogun

Abdulrahman

Majid Erfanian

Fikri

Yunzhu Gao

Mustafa Gurbuz

Madan Mohan Panja

Francisco Bulnes

Ahmad Hamza Al Cheikha

Mehmet 